\title{
Consumo de prácticas culturales en jóvenes universitarios de algunos países latinoamericanos*
}

\author{
Consumption of Cultural Practices among College Young People in some Latin-American Countries Consumo de \\ práticas culturais em jovens universitários de alguns países latino-americanos
}

Clara Inés Villamil Guzmán ${ }^{a}$

Universidad de Boyacá, Colombia

clavillamil@uniboyaca.edu.co

ORCID: http://orcid.org/0000-0003-4838-9564

\section{Luz Marina Hurtado Torres}

Universidad de Boyacá, Colombia

ORCID: http://orcid.org/0000-0002-0046-7132
DOI: https://doi.org/10.11144/Javeriana.syp38-75.cpcj

Fecha de recepción: 07 Noviembre 2018

Fecha de aprobación: 30 Mayo 2019

Fecha de publicación: 15 Noviembre 2019

\section{Resumen:}

El artículo tiene como objetivo hacer una revisión de estudios sobre el consumo de prácticas culturales en jóvenes de algunas universidades latinoamericanas. Se entiende como práctica cultural las actividades referentes a medios escritos y audiovisuales, museos, exposiciones, deportes virtuales, tecnologías de la información y la comunicación. Se analizaron 52 investigaciones de artículos, revistas indexadas, tesis e informes, realizados entre los años 2001 y 2018, en los cuales se presentan los avances y vacíos en esta temática. Los análisis de estos estudios permiten concluir que el consumo cultural de los jóvenes universitarios se transformó como consecuencia de la globalización, el avance de las tecnologías de la información y comunicación, y la influencia de las nuevas industrias culturales.

Palabras clave: consumo cultural, jóvenes universitarios, universidades latinoamericanas, prácticas culturales.

\section{Abstract:}

This article aims to review the studies on the consumption of cultural practices among the youth in some Latin-American colleges. A cultural practice is understood herein as any activity related to written and audiovisual media, museums, exhibitions, virtual sports, information technology and communication. Fifty-two researches conducted between 2001 and 2018 and published as articles, indexed journals, degree projects and reports were analyzed. They showed both the advances and gaps on this topic. The analyses of these studies allow concluding that the cultural consumption by the college youth has been transformed due to the globalization, the ICT advancement, and the influence of the new cultural industries.

Keywords: cultural consumption, college young people, Latin-American universities, cultural practices.

\section{Resumo:}

O artigo objetiva fazer revisão de estudos sobre o consumo de práticas culturais em jovens de algumas universidades latinoamericanas. Entende-se como prática cultural as atividades referentes a mídia escrita e audiovisual, museus, exposições, esportes virtuais, tecnologias de informação e comunicação. Foram analisadas 52 pesquisas de artigos, revistas indexadas, teses e relatórios, realizados entre os anos 2001 e 2018, nos quais houve avanços e lacunas nesta temática. As análises desses estudos permitem concluir que o consumo cultural dos jovens universitários mudou em consequência da globalização, o avanço das tecnologias da informação e comunicação e a influencia das novas indústrias culturais.

Palavras-chave: consumo cultural, jovens universitários, universidades latino-americanas, práticas culturais.

Notas de autor:

a Autora de correspondencia. Correo electrónico: clavillamil@uniboyaca.edu.co 


\section{Introducción}

En la actualidad, los estudios sobre el consumo de prácticas culturales en espacios educativos de nivel superior son objeto de análisis en el escenario académico. Es decir que el consumo cultural ha dejado de ser un campo exclusivamente económico para ser considerado como objeto de estudio en el ámbito de lo social, de lo cultural y de lo simbólico, como lo plantea Bourdieu (1998).

Por su parte, Hinojosa (2012) define las prácticas culturales como

aquellas actividades donde incluyen, a un gran número de población. Entre estas se consideran conciertos, televisión, cine, radio, deportes, cursos alternativos, monumentos, museos, y el consumo de otras ofertas culturales como espectáculos de circo, teatro, y danzas, además de la lectura de libros, asistencia a bibliotecas, exposiciones, entre otras. (p. 6)

Las nuevas dinámicas mundiales -entre estas, la globalización, las nuevas tecnologías, y las industrias culturales- están produciendo transformaciones en el consumo de las prácticas culturales en los jóvenes universitarios, lo que hace que la utilización de algunas de estas sea cada vez más diversa y llevada a cabo con diferente frecuencia, mientras que otras están dejando de ser consumidas.

Bajo esta perspectiva, el objetivo del siguiente trabajo es analizar las investigaciones realizadas sobre el consumo de prácticas culturales en estudiantes universitarios de algunos países latinoamericanos. Se busca conocer acerca de su vida cultural mediante las siguientes preguntas: ¿qué prácticas culturales realizan en su tiempo libre?, ¿ cuáles comparten entre ellos?, ¿qué aspectos inciden en el consumo de estas? y ¿cómo estas se transforman en medio de una sociedad globalizada, mercantilizada, individualizada y en constante cambio?

La revisión y el análisis de las investigaciones se hizo con base en los siguientes criterios de inclusión: consumo cultural de medios escritos y audiovisuales, museos y exposiciones, consumo de tecnologías de la información y la comunicación, realizados entre los años 2001 y 2018, en jóvenes de todos los programas académicos de universidades públicas y privadas de México, Argentina, Chile, Cuba, Ecuador, Colombia, Perú, Panamá, Uruguay y Bolivia.

\section{Medios escritos: lectura de libros, periódicos y revistas}

La investigación de Hinojosa, titulada "Consumo y prácticas culturales de la comunidad universitaria de una universidad mexicana”, la Universidad Autónoma de Nuevo León, muestra que los universitarios presentan un bajo consumo cultural de libros y de asistencia a bibliotecas, "un $22 \%$ de los jóvenes lee dos libros al año. Estos resultados indican un nivel de lectura poco satisfactorio. Más de la mitad de las personas no acostumbran a ir a la biblioteca o casi nunca 54,6\%" (Hinojosa, 2012, p. 8). En el contexto nacional mexicano, la Encuesta Nacional de Lectura realizada por el Consejo Nacional para la Cultura y las Artes (Conaculta) -en la cual se incluye universitarios- devela que "la población mexicana que reporta tener un mayor índice de lectura se encuentra entre los jóvenes de 18 y 22 años de edad, lo cual corresponde al 68\% de universitarios" (Conaculta, 2006, p. 11).

En el artículo "Prácticas culturales en jóvenes universitarios cubanos", realizado en la Universidad de Granma, Gómez y Riverón (2017) indican que

aunque si bien el 90\% de los jóvenes encuestados sienten motivación por la lectura, denota una preocupación el rango de prioridad o jerarquía al tipo de lectura, por ejemplo, las novelas, las aventuras, las revistas representan el $70 \%$, mientras que, los géneros históricos, políticos o sociales, solo el 20\%, los cuales son de vital importancia para la formación profesional. (p. 108)

Los anteriores estudios realizados en las universidades de México y Cuba demuestran que hay distintos propósitos por la práctica cultural de la lectura entre los jóvenes. Por su parte, en la universidad mexicana se presenta un bajo nivel de consumo de lectura. En la universidad cubana de Granma, dicha práctica cultural es diversificada, aunque se presenta un reducido consumo de lectura. Mientras que en la Universidad de Buenos 
Aires y en la Universidad de Ciencias Empresariales y Sociales en Argentina, el estudio de Ravettino (2012), titulado "Prácticas de lectura. Acerca del placer y la literatura en jóvenes", muestra un elevado nivel de libros leídos al año, y los aspectos que inciden en su realización y en el gusto por dicha lectura, "los lectores que tienen el hábito de leer lo hacen por placer, es decir, en cuanto a lectura vinculada con el entretenimiento leen entre 1 y 5 libros anuales, que corresponden al 50\%” (p. 9).

En los jóvenes universitarios chilenos de la Región Metropolitana, adscritos a universidades privadas, Gilardoni, en su investigación "Valoración del libro y mecanismos de acercamiento a la lectura en los estudiantes universitarios", revela la importancia que tiene el consumo de lectura: "un $91 \%$ de los encuestados se refiere a dicha actividad de un modo positivo bajo las opciones: un agrado, una necesidad para complementar mis estudios y una alternativa más de entretenimiento" (Gilardoni, 2006, p. 24).

La investigación realizada por Yuquilima en Ecuador, denominada "Estudio del insuficiente hábito en la lectura de los estudiantes de la Universidad de Guayaquil", llama la atención por el bajo consumo cultural de la lectura en los jóvenes que se encuentran en los primeros semestres de la universidad, "el 55\% de estudiantes no les gusta leer, el 33\% lee medianamente, el 12\% de estudiantes les gusta leer” (Yuquilima, 2015, p. 113).

El estudio realizado por De la Puente en la Universidad La Molina y Villa El Salvador, titulado "Motivación hacia la lectura, hábito de lectura y comprensión de textos en estudiantes de psicología de dos universidades particulares de Lima Perú", indica que si bien hay interés en el consumo de lectura, se presentan bajos niveles en comprensión: "un 96,7\% no comprende lo que lee, a pesar de que un alto porcentaje de encuestados: 82,42\%, se encuentra en el nivel medio de la escala de motivación” (De la Puente, 2017, pp. 71-72).

El trabajo de Callejas, "Estilos de vida universitaria de los estudiantes de la Universidad Mayor de San Andrés en Bolivia", presenta una situación similar referente al bajo consumo cultural de libros, aunado a la poca asistencia a las bibliotecas: "asistir a la biblioteca a leer, a investigar, es un aspecto que presenta menor recurrencia. Los pocos estudiantes que van a la biblioteca, van a realizar trabajos o a conversar" (Callejas, 2016, p. 39).

El estudio de Andrade, "La lectura en los universitarios. Un caso específico: Universidad Colegio Mayor de Cundinamarca - Colombia", devela el mínimo consumo de libros,

el 23\% de los jóvenes lee en el año entre dos y tres libros. Sobre lo cual infiere que, si bien tienen cierto grado de conciencia por la lectura, prefieren escuchar música, aunque consideran que leer es una necesidad, lo hacen en forma esporádica los fines de semana. (Andrade, 2007, p. 236)

Por su parte, Rodríguez, Solano, Martínez y del Villar, en la investigación que llevaron a cabo en las universidades colombianas, Autónoma de Occidente, Católica Popular de Risaralda, Central del Valle, Universidad de Ibagué, Universidad del Quindío, Pontificia Universidad Javeriana Cali, Universidad Pedagógica Nacional, Universidad del Cauca, Universidad de la Amazonía, Universidad de Córdoba, Universidad del Atlántico, Universidad de Antioquía, Pontificia Universidad Javeriana de Bogotá, Universidad del Valle y Universidad de Caldas, muestran que

los alumnos tienen como propósito en sus actividades de lectura y escritura, casi que exclusivamente, responder por sus responsabilidades académicas en sus asignaturas. Los documentos que leen, son el $80 \%$ los apuntes tomados de las clases, mientras que los documentos elaborados por los profesores, libros propios de la asignatura, informes periodísticos e informes de investigación, literatura y novelas ocupan los últimos lugares en documentos leídos. (Rodríguez, Solano, Martínez y del Villar, 2013, p. 14).

El estudio realizado en Villavicencio, Colombia por Chaparro y Guzmán, denominado "Jóvenes y consumo cultural. Una aproximación a la significación de los aportes mediáticos en la preferencia juveniles”, describe que

los jóvenes tienen una frecuencia de lectura de libros de una vez a la semana 35,6\% o; leer novelas el 43,7\% por encima de cuentos que es el 36,6\%, poesía 15,2\% o libros de autoayuda el 9,1 \%. El interés de los jóvenes en la lectura está relacionado con un pasatiempo, seguido por expectativas educativas 25,4\% informativas 25,8\%. (Chaparro y Guzmán, 2017, p. 135) 
La investigación de Huertas, Caro, Vásquez y Vélez (2008), "Consumo cultural y uso del tiempo libre en estudiantes de la Universidad de la Salle - Colombia”, revela que

La lectura de la prensa escrita aparece como una actividad de mediana intensidad. De acuerdo con lo anterior, podemos establecer tres niveles de lectura: un nivel bajo, los que rara vez ojean un periódico $45,8 \%$; un nivel medio, agrupa a los que leen entre una y tres veces por semana 40,2\% y un nivel alto, los que leen la prensa 4 veces y más el 14,0\%. (Huertas et al., 2008, p. 40)

De acuerdo con los hallazgos en estas investigaciones, se evidencia que el mayor consumo de libros se hace por jóvenes universitarios en Argentina, Chile y Cuba; mientras que en Bolivia, Perú y Ecuador, los índices de consumo cultural de lectura son bajos. Uno de los motivos para explicar este bajo consumo es la baja comprensión de lo que se lee. Para el caso colombiano, se muestra que los jóvenes, en un alto porcentaje, solo leen apuntes tomados en clase.

\section{Museos y exposiciones}

La investigación realizada por Hinojosa en la Universidad Autónoma de Nuevo León México sobre el consumo de museos muestra que "el 52\% de la población visitó un museo en los últimos 12 meses y, un $43 \%$ visitó monumentos, lugares históricos o arqueológicos, la identidad tiene un papel importante en dicho consumo" (Hinojosa, 2012, p. 11). Nivón y Sánchez, en su artículo "Algunas consideraciones sobre los estudios de consumo cultural en México y en Chile", observan el elevado consumo de esta práctica cultural: “en México es notable la asistencia a museos” (Nivón y Sánchez, 2012, p. 66).

En la Universidad de Granma en Cuba, Gómez y Riverón realizaron el estudio "Prácticas Culturales en jóvenes universitarios cubanos", en el cual hallaron que "hay indiferencia, desinterés, por ofertas de los centros culturales, relativo a visitas, museos, exposiciones" (Gómez y Riverón, 2017, p. 102). Ahora bien, AntoineFaúndez y Carmona-Jiménez, en el trabajo realizado en la Universidad del Pacífico, "Museos y jóvenes: entre la incomprensión y el desencanto. Percepciones y argumentos juveniles sobre el consumo cultural de museos en Chile", muestran que "el 73,8\% de la población chilena no asiste a museos" (Antoine-Faúndez y CarmonaJiménez, 2015, pp. 230-231).

En Perú, Alata (2013), en el Informe del observatorio ciudadano "Lima cómo vamos", devela que "el 27,8\% de limeños visitó museos, monumentos, lugares históricos y arqueológicos" lo cual evidencia "la baja asistencia a este tipo de lugares" (p. 4). El estudio de Andrade, "Determinantes de la asistencia a museos en Colombia. Una perspectiva desde la economía de la cultura", señala que "el $87,4 \%$ no asiste a los museos y tan solo un $12,6 \%$ asiste a estos espacios culturales" (Andrade, 2016, p. 239). Por su lado, el ya mencionado trabajo de Chaparro y Guzmán, "Jóvenes y consumo cultural. Una aproximación a la significación de los aportes mediáticos en las preferencias juveniles", muestra la baja asistencia a museos y exposiciones: "un 39,6 \% nunca lo han hecho por razones asociadas a la escasa oferta, 47,3 \%, la falta de divulgación 15,9\% y desinterés 12,3 \%” (Chaparro y Guzmán, 2017, p. 134).

De acuerdo con las investigaciones, se muestra que el mayor consumo de museos se presenta entre los jóvenes universitarios mexicanos, lo cual difiere con el bajo consumo presentado en las universidades de Chile, Colombia, Perú y Cuba, y muestra que estas prácticas culturales han perdido la importancia y el interés en esta población.

\section{Teatro, cine y danza}

Con respecto al consumo de teatro, cine y danza, Chaparro y Guzmán señalan que: 
un alto porcentaje afirmó no asistir a este tipo de actividades 47,3\% o hacerlo con poca frecuencia 42,2\%. Esto es significativo, ya que en sumatoria el $89,5 \%$ de los encuestados manifiesta que con muy poca frecuencia o nunca asiste a cine o al teatro. Esto se relaciona con la escasa o nula difusión de las presentaciones artísticas en el área de teatro y el poco interés que se tiene en la región por desarrollar propuestas que acerquen a los jóvenes a sus prácticas culturales preferidas. (Chaparro y Guzmán, 2017, p. 135).

Por su parte, Huertas et al. (2008) encontraron que "un porcentaje considerable 65,8\% asisten al cine por lo menos una vez cada dos o tres meses, habiendo un $28 \%$ que frecuenta las salas de cine por lo menos una vez al mes y un $18,2 \%$ que asiste semanal o quincenalmente" (p. 40).

"Lima cómo vamos", realizado en Perú en 2013, indica que

la principal actividad cultural realizada por los limeños es "ir al cine”, con un 56,5\% de asistencia. Esta es la segunda actividad más frecuente, donde "ir a pasear a parques" ocupa el primer lugar con el 79,3\%. Por otro lado, ha habido una ligera disminución en la participación en eventos culturales en las calles, ir a ferias del libro o artesanales y asistir a muestras o encuentros gastronómicos a comparación del año 2010. (Alata, 2013, p. 5)

El trabajo de Gamboa y Rivera Viera, denominado "Jóvenes universitarios: relación con el arte, la cultura y la tecnología en la Universidad Javeriana Cali", muestra que "entre las mujeres el baile favorito es la salsa seguido ballet, y con menos preferencia Capoeira, las danzas folclóricas, aparecen con muy poco interés" (Gamboa y Rivera Viera, 2015, p. 81). El estudio de Arroyo, titulado "Consumo cultural en jóvenes estudiantes de pregrado de la pontificia Universidad Católica del Perú", devela que "el consumo de cine es de un $45 \%$ en el cual asisten todas las semanas, y solo un 4\% no asisten" (Arroyo, 2011, p. 39).

En concordancia con Bourdieu, frente al consumo por los estratos socioeconómicos establecidos por el capitalismo, Arroyo señala que: “desde la clase media 'para arriba', al tener un poder adquisitivo mayor, son las personas que tienen dinero para invertir en ocio. [...] Sin embargo, el gran público es la clase media. Particularmente, los jóvenes” (Arroyo, 2011, p. 39).

El trabajo de Quintar y Borello, "Consumos culturales en Argentina: El caso del cine en la población de estudiantes universitarios" - realizado con alumnos de universidades públicas de las ciudades de Buenos Aires, Córdoba, Mendoza, Rosario, Bariloche, Neuquén- devela que: "el primer lugar es la TV por cable, siguiéndole en orden decreciente las salas de cine y, en tercer lugar, los DVD no originales que se venden en la calle" (Quintar y Borello, 2014, pp. 8-9). Así mismo, el estudio de Sandoval muestra que "una forma de participación social de tipo cultural, ofertadas por la universidad y en las que se involucran los jóvenes son el coro la danzas y el teatro con un 12,3\%" (Sandoval, 2016, p. 179).

El trabajo de Portocarrero, titulado "Estudio sobre el consumo cultural de los universitarios de la Universidad Nacional Mayor de San Marcos -Perú", indica que "el 2\% de los estudiantes tiene un nivel bajo de asistencia a presentaciones y espectáculos culturales, el 30\% un nivel medio y el 68\% indicaron nivel alto de asistencia" (Portocarrero, 2017, p. 56).

\section{Televisión}

El estudio de Ríos, Matas y Gómez, titulado "Investigación sobre el uso de la televisión por estudiantes de educación de universidades hispanoamericanas" - el cual fue realizado en las universidades públicas de Catamarca y Nacional de Cuyo en Argentina; la Universidad Autónoma de Ciudad Juárez y Guadalajara, sede de CuValles en México; la Universidad de Guadalajara y la Universidad Virtual México; la Universidad Santo Tomas de Chile y la Santo Toribio de Mogrovejo Perú, en alumnos de los programas de Ciencias de la Educación- muestra que hay "una tendencia a un uso y opinión sobre los medios estudiados que no depende del sexo, edad, país de residencia, universidad en la que se estudia, o modalidad de estudios elegido, lo que indica que se está produciendo una homogeneización, respecto al uso de la televisión" (Ríos, Matas y Gómez, 2011, p. 321). 
Por su parte, el trabajo de Paredes y Behn indica que

según el género las mujeres prefieren más que los hombres las siguientes actividades: ver TV o escuchar música 92,8\% versus $84,9 \%$, leer $20,2 \%$ versus $9,2 \%$. Las actividades preferidas por los hombres más que las mujeres son: practicar deportes $32,8 \%$ versus $4,3 \%$, salir a restaurante $27,7 \%$ versus $17 \%$, juegos computacionales $20,2 \%$ versus $13,6 \%$ e ir a eventos deportivos o cine $11,8 \%$ versus $5,6 \%$. (Paredes y Behn, 2008, pp. 58-59)

El estudio de Cabanzo, "Ocio y tiempo libre: una aproximación a sus representaciones y percepciones en los estudiantes de la Universidad Pedagógica Nacional Bogotá, encontró que "el 91,8\% escucha música; leer y escribir $81,5 \%$; conversar con amigos $82,4 \%$, realizar actividades culturales el $28,3 \%$ y usar videojuegos el 26\%" (Cabanzo, 2009, p. 16).

Arango y González, en su estudio realizado en la Universidades de La Sabana, Universidad Nacional de Colombia, Universidad Pontificia Bolivariana, Universidad Autónoma de Occidente, Universidad de Antioquia, Universidad de los Llanos, Fundación Universidad del Norte y Universidad de Manizales, devela que "los contenidos televisivos preferidos por los jóvenes son películas y videos musicales, los realities y los concursos no tienen buena aceptación. Los programas de actualidad, los documentales y los noticieros son los contenidos favoritos" (Arango y González, 2009).

Ahora bien, el estudio de Huertas et al. (2008) indica, con respecto a los jóvenes, que en la Universidad de la Salle, "29\% de lunes a viernes ve televisión durante 20 horas y un 36,8\% entre cinco y diez horas semanales. Los fines de semana el consumo de televisión no disminuye. El estudiante le dedica entre 30 y 35 horas semanales al consumo televisivo" (p. 41).

El trabajo de Chaparro y Guzmán enseña que, en cuanto a:

programas de televisión preferidos, los jóvenes manifiestan en un $36 \%$ de favoritismo por novelas; seguido por programas de comedia un $25,4 \%$ y de acción un $22,8 \%$. La ciencia ficción ocupa el 14,9\% y los realities el 14,7\%. Los programas informativos y noticiosos no atraen la atención de los jóvenes por ser considerados muy serios. (Chaparro y Guzmán, 2017, p. 138)

Los estudios de Huertas et al., llevados a cabo en la Universidad de la Salle - Colombia, señalan sobre el consumo de radio que "el 69\% de los estudiantes dedica parte de su tiempo a escucharlo" (Huertas et al., 2008, p. 40).

El trabajo de Suing, Ordóñez y Ortiz, "El consumo de radio y televisión de los estudiantes universitarios del Ecuador”, realizado en jóvenes de las universidades Central del Ecuador, De las Américas, De los Hemisferios, Estatal de Bolívar, De Cuenca, Iberoamericana, Internacional del Ecuador, Metropolitana UMET, Nacional de Loja, San Gregorio de Portoviejo, Regional Amazónica Ikiam, y San Francisco de Quito, muestra que "el tiempo dedicado por día para ver televisión a través de Internet fluctúa entre menos de una hora $42 \%$, una hora 22\%, dos 25\%, tres 7\% y más de tres horas 4\%” (Suing, Ordóñez y Ortiz, 2018, p. 12).

El trabajo de Pérez y Castro (2015), titulado "Jóvenes en la encrucijada de los nuevos tiempos. Una mirada a sus prácticas y consumos culturales desde la Universidad de Granma - Cuba”, muestra que el 90\% ve televisión como una de las actividades que realiza en su tiempo libre

El estudio de Casillas, Molina y Colorado (2011), denominado "Usos del tiempo de los estudiantes de la Universidad de Veracruz en México”, muestra también el elevado consumo de televisión cuatro horas diarias dedican a ver televisión. El trabajo de Munguía (2006), titulado "Consumo de medios de comunicación y la construcción de la imagen de los partidos jóvenes universitarios", revela que el 70\% de los estudiantes de Lima dan privilegio a la televisión. 


\section{Radio}

El estudio de Vergara, Cerezo, Cifuentes, Nieto y Parra (2009), titulado "Consumo de medios masivos de comunicación en estudiantes universitarios de Manizales”, señala que el 67\% de los estudiantes escucha radio, convirtiéndose en uno de los medios más escuchados por esta población con sus programas radiales.

La investigación de Caro y Vásquez, "Consumo cultural y uso del tiempo libre en universitarios del área metropolitana - Medellín", evidencia que "el 45\% de los universitarios escuchan la radio de una a cuatro horas diarias, un 13\% no la oyen” (Caro y Vásquez, 2012, p. 16).

El trabajo de Acosta y otros, denominado "Radio cultural: estudio del consumo de los estudiantes y trabajadores de la Universidad de Colima - México", indica que "de los estudiantes el 86\% escucha radio y el $14 \%$ no. Teniendo mayor inclinación a la radio comercial con un $71 \%$ y un 29\% la radio cultural" (Acosta et al., 2014, p. 187).

\section{Música}

Con respecto a la música, el trabajo de Juni, “Consumo musical de los jóvenes uruguayos", realizado en la Universidad de la República, señala que "los estudiantes acordaron que existe un mayor consumo de artistas internacionales, así como también de géneros que no son considerados como propios de nuestro país" (Juni, 2015, p. 29). La investigación de Terrazas, Lorenzo y González (2013), denominada "Consumo musical de estudiantes universitarios de México. Una comparación entre alumnos de distintas facultades de la Universidad Autónoma de Chihuahua", muestra el consumo de géneros musicales pop (12,8\%), banda norteña $(12,1 \%)$ y rock $(11,3 \%)$ como aquellos de mayor preferencia por los jóvenes de las Facultad de Derecho, Facultad de Economía Internacional y la Facultad de Educación Física. Otros géneros escuchados son música clásica, metal, jazz, corridos, y country por estudiantes de la Facultad Artes.

\section{La tecnología y el Internet: transformaciones en el consumo cultural}

El fenómeno de estar conectado a las redes sociales es lo que Castells (2007) denomina en su estudio Comunicación Móvil y Sociedad, como sensibilidad tecnosocial, es decir,

el estado en el que la tecnología y la naturaleza se unen. El concepto de tecnosocialidad pone énfasis en las tecnologías de la comunicación, no como herramientas, sino como contextos, condiciones ambientales que hacen posible nuevas maneras de ser, nuevas cadenas de valores y nuevas sensibilidades sobre tiempo, espacio y los acontecimientos culturales. (p. 226)

Es así que el consumo del lenguaje digital, Castells (2007) lo considera como:

consolidación de los grupos de iguales en función de valores compartidos y códigos de significado, para los miembros del grupo lleva al surgimiento de la identidad colectiva. La(s) cultura(s) juvenil(es) están marcadas por presencia de estos códigos; por ejemplo, una lengua compartida, como en la práctica de mensajes de texto en la comunicación inalámbrica, y en la adopción de nuevas formas de expresión de la lengua escrita. Esta es una forma en que la evolución tecnológica actúa como factor de cambio cultural y de comportamiento en la sociedad móvil. (p. 229)

La globalización ha incidido en el consumo de las tecnologías, como lo plantea Castells: "la globalización es un proceso cultural en marcha que forma parte íntima de nuestra sociedad. Está globalizada también, en cierto modo, la comunicación a través de la formación de grandes conglomerados en medios de comunicación" (Castells, 2000, p. 46). Estas nuevas dinámicas en los consumos culturales tecnológicos son analizadas desde diferentes perspectivas. El trabajo de Durand, Bombelli, Barberis y Bouzón (2009), "Representaciones y modo de uso de nuevas tecnologías de información y comunicación entre estudiantes 
universitarios de Buenos Aires Argentina", explica que "el 96\% de los estudiantes encuestados utiliza Internet, para realizar negocios, comprar, buscar información de cualquier tipo, realizar trabajos de la facultad, comunicarse en el trabajo, con amigos y familiares" (p. 6).

En la Universidad Autónoma de Nuevo León México, el estudio de Hinojosa indica que "en este ámbito son los videojuegos, los cuales usan un $40 \%$ del alumnado. Las plataformas más utilizadas son: Play Station con un 25,7\%, el Xbox con un 22\%" (Hinojosa, 2012, p. 13).

De igual manera, el trabajo de Callejas en la Universidad Mayor de San Andrés en Bolivia plantea que "el $81 \%$ de los estudiantes tiene alto interés en mantenerse conectados al internet, acceden a redes sociales o de entretenimiento ya que se puede descargar juegos, música, videos, etc." (Callejas, 2016, p. 39).

En "El tiempo libre de los estudiantes universitarios de la Facultad de Comercio y Administración de Tampico de la Universidad Autónoma de Tamaulipas en México", se manifiesta que "los universitarios invierten parte de su tiempo libre en conversar en redes sociales ver televisión y deportes" (Rangel y Ochoa, 2012, p. 24)

El trabajo de Ortega, "El impacto de las nuevas tecnologías digitales en la cultura comunicativa de los jóvenes universitarios una visión panameña” - realizado en la Universidad Santa María, la Universidad Antigua, la Universidad de Panamá y la Universidad Tecnológica de Panamá-, muestra el elevado índice de consumo de Internet,

el 93,8\% usa internet para realizar tareas universitarias, el 74,3\% para entretenimiento, el $72 \%$ para chatear, en tanto que un $62,3 \%$ dijo que para interactuar en las redes sociales. Buscando explorar y conocer cómo manejan los jóvenes su capacidad afectiva, mediada por la tecnología, en la encuesta se preguntó sobre el nivel socioafectivo a través de las redes sociales. A este respecto, el 37\% dijo que las relaciones en esas plataformas son fáciles de mantener; y un 30,4\% dijo que son afectivas, y solo un $17 \%$ dijo que son superficiales. (Ortega, 2012, pp. 78-79)

La investigación de Basantes, Naranjo, Gallegos, Grijalva y Villarreal (2016), "Hábitos de consumo y uso de medios digitales en los estudiantes de la Universidad Técnica del Norte, Ecuador" señala que

el principal medio que usan los estudiantes universitarios para conocer las novedades tecnológicas es la internet con un $96 \%$. La red social con mayor alcance entre los estudiantes es Facebook. En segundo lugar, de frecuencia se encuentra Youtube. Entre los servicios de mensajería instantánea, se observa mayor uso de Whatsapp. (Basantes et al., 2016, p. 26)

La investigación llevada a cabo en la Universidad de Ibagué por Puerta y Carbonell muestra el alto consumo de internet: "el promedio de horas de uso diario de Internet es de 4,56\%; el 4,2\% dedicaba más de siete horas a Internet. Las aplicaciones más usadas son las redes sociales $84 \%$, el correo electrónico $50,4 \%$ y los motores de búsqueda 43,1册0. La red social preferente fue Facebook 83,3\%" (Puerta y Carbonell, 2013, p. 624). El estudio de Huertas et al. (2008) estableció que en la Universidad de la Salle, sede Medellín,

el 40,8\% dedica tres horas a navegar en la red, el 21,9\% cuatro horas y el 20\% dos horas. En promedio los estudiantes hacen uso de la Internet durante 21 horas a la semana, un 5,4\% le dedica más de cinco horas diarias al Chat, que daría en total un número de 35 horas semanales. (p. 41)

"Los jóvenes y la red: usos y consumos de los nuevos medios en la sociedad de la información y la comunicación", realizada en la Universidad Jorge Tadeo Lozano, muestra los diferentes usos que tiene la internet entre los jóvenes universitarios:

existen múltiples usos del computador por parte del estudiante; prima el uso del chat, el correo y el Messenger. El uso del computador y la conexión a Internet se dan diariamente. Les parece "caótica" la vida sin Internet la red es un mecanismo que permite acceder a los datos actualizados de entretenimiento, ocio, entre otros; los jóvenes se actualizan, a través de la red, en tecnología y diversión (juegos); Internet propone guías de comportamiento social para seguir y es un mecanismo que les permite darse a conocer y conocer a los demás. (Barrios, 2009, p. 272).

El estudio de Chaparro y Guzmán (2017) describe que: 
el porcentaje de jóvenes que tiene acceso a internet es un 51,6\%, para entretenimiento un 54,2\%; con fines informativos un $32,9 \%$ o educativos $24,6 \%$. Al mostrar la preferencia respecto a las páginas y portales: en primer lugar, aparece Facebook 50\%, y en menor medida la mensajería instantánea Whatsapp con $27,3 \%$ y el portal especializado en música y vídeo YouTube un $34,7 \%$ y están adscritos a redes sociales un 69,1\%. (Chaparro y Guzmán 2017, p. 137)

El trabajo de Rodríguez, "Redes sociales y hábitos de consumo en estudiantes universitarios, caso Universidad Pedagógica y Tecnológica de Colombia”, muestra las diferencias en el consumo cultural de redes sociales entre mujeres y hombres de pregrado. La red Instagram es más utilizada por mujeres, y presenta apatía para los hombres. Por su parte, Facebook es la red social de mayor uso y frecuencia entre mujeres con un 47,4\%, y hombres con un $45,9 \%$. El segundo lugar es ocupado por el email, seguido de Instagram y WhatsApp, que representan un porcentaje de uso importante. Finalmente, Twitter es usado en menor proporción (Rodríguez, 2018).

La investigación de Dávila, Rivera y Chávez, "Usos del tiempo y consumo cultural: Caracterización de estudiantes de una Universidad Rural del Sur del Estado de Sonora - México”, muestra que el uso de la computadora y el Internet reportan un uso muy similar, representando alrededor de ocho de cada diez estudiantes. Las actividades que realizan al conectarse a Internet son: "bajar información, uso de correo electrónico, consultar material especializado, chatear y mantenerse en contacto con amigos y conocidos en Facebook. Consultar material especializado y almacenar/compartir información” (Dávila et al., 2017, p. 11).

Cabrera et al. (2017) develan que el consumo de medios electrónicos como el internet es alto, "el 20,5\% lo consume en la Universidad de Guadalajara y un 13,8\%, en la Universidad de Guatemala” (p. 35). La investigación de Aguilar y Ramírez, "Hábitos de consumo de las tecnologías de la información en los estudiantes universitarios de Tijuana - México", muestra que "el 30\% del tiempo que se está conectado al internet se ocupa en chateo. El 21,2\% lo ha usado para realizar compras por internet" (Aguilar y Ramírez, 2007, p. 13).

La tesis doctoral de Sánchez, "Prácticas de consumo audiovisual en internet en estudiantes universitarios de la ciudad de Medellín (Upb, Udea, Politécnico Jic, Udem, Eafit)", menciona que "el consumo de contenidos en internet corresponden a ver películas con un $88 \%$, videos musicales el $82 \%$, cortometrajes el $54 \%$, series televisivas el 53\%, documentales el 46\% y videos de humor 43\%” (Sánchez, 2013, p. 89).

El trabajo de Nobles et al. (2016) "Tecnologías de la comunicación y relaciones interpersonales en jóvenes universitarios en la Universidad Cooperativa de Colombia, sede Montería, indica que los estudiantes consideran que

invierten demasiado tiempo en el uso de tecnologías, representado por el 64\%. La red social utilizada con mayor frecuencia entre los estudiantes es Facebook, usada por el 37\%; de igual forma, el 32\% de los mismos elige a Instagram como otra red social de su preferencia. También se encontró que el 52,3\% de los estudiantes encuestados afirma haber desarrollado nuevas habilidades de comunicación en su interacción social gracias a la utilización de las diversas redes sociales, el encontrar amigos que hacía tiempo no veían, estar en contacto constante con familiares y amigos, compartir fotos, experiencias, todo lo cual ha permitido el fortalecimiento de sus relaciones sociales. (Nobles et al., 2016, p. 23).

"Consumo y uso de medios de comunicación de jóvenes universitarios ecuatorianos en tiempos de convergencia" muestra que "el consumo del teléfono móvil en un $72 \%$, acceder a internet y redes sociales un 61,55\%, lo cual corresponde a 6 de cada 10 jóvenes; escuchar música 5\%, enviar e- mail 5,7\%” (Velásquez, 2015, p. 169).

La investigación sobre deportes virtuales, desarrollada por Marcano, considera a este tipo de consumos tecnológicos como

e-sport o deportes electrónicos a las prácticas de videojuegos con fines competitivos a través de los que se obtiene dinero como premio para los que alcanzan los primeros lugares en las competiciones. Incluye a los equipos o videojugadores que tienen un salario mensual por ser jugadores "profesionales". Estos en general son pagados por las grandes corporaciones productoras de videojuegos. (Marcano, 2012, p. 116) 
La investigación de Alarcón y Larraz señala que de acuerdo al consumo de la tecnología surgen los nativos digitales, como los denominados Millennials, que son

la Generación de los nacidos entre 1981 y 1995, jóvenes entre 20 y 35 años que se hicieron adultos con el cambio de milenio. Actualmente en Latinoamérica un 30\% de la población es Millennial. Se estima que para el 2025 representarán el 75\% de la fuerza laboral del mundo. Se caracterizan por dominar la tecnología como una prolongación de su propio cuerpo. Casi todas sus relaciones cotidianas están intermediadas por una pantalla. Para ellos, realidad y virtualidad son dos caras de la misma moneda. Prefieren internet a la TV convencional. (Alarcón y Larraz, 2015, p. 14).

Los estudios demuestran que el consumo cultural de tecnologías de la información y la comunicación, como el Internet, presenta un alto porcentaje entre los universitarios, ya que son usados para fines sociales, culturales, académicos y recreativos. Es así que estos no son solo un medio de comunicación, sino una parte de su vida social.

En estos consumos culturales tecnológicos, hay que tener en cuenta los factores que influyen. Según Warnier, parte de estos son los medios de comunicación y el sistema económico, ante lo cual señala que "la actividad de las industrias culturales y de los medios no se sostiene a largo plazo sino respetando lógicas económicas, las empresas incrementan sus ingresos vendiendo espacios o tiempos a anunciadores publicitarios o vendiendo productos culturales" (Warnier, 2001, p. 49).

\section{Conclusiones}

Los diferentes estudios sobre el consumo de prácticas culturales en jóvenes universitarios de todos los programas académicos, y de universidades públicas y privadas de México, Argentina, Chile, Cuba, Ecuador, Colombia, Perú, Panamá, Uruguay y Bolivia, muestran que, en cuanto al consumo de libros, los universitarios de Argentina y Chile llevan a cabo esta práctica cultural por gusto, y en un alto porcentaje. El caso de Cuba es particular, pues allí también se tiene un elevado nivel de lectura en todos los géneros, tanto científicos como literarios.

Por su parte, en los estudiantes de las universidades de México, Ecuador y Bolivia se evidencia que esta práctica cultural de la lectura de libros y otros textos presenta poco consumo, y cuando se realiza tiene fines principalmente académicos. En el caso de Colombia, los estudiantes leen prácticamente solo los apuntes registrados en clase, lo cual muestra que esta práctica es cada vez más abandonada entre los universitarios. El caso de Perú es interesante, si bien hay interés por el consumo de libros, hay bajos niveles de comprensión y análisis de lo leído, lo cual afecta su desempeño académico y profesional. Algo similar sucede en Bolivia, donde se asiste a las bibliotecas para hacer otras prácticas académicas diferentes a las de la lectura.

En lo relacionado al consumo cultural colombiano de museos, teatro, radio, cine y danza, estas prácticas son cada vez más abandonadas, debido a que los jóvenes no las encuentran llamativas. Además, hay poca difusión de los eventos, los lugares donde se llevan a cabo se encuentran alejados de su vivienda, y sumado a esto está la falta de dinero. Sin embargo, en los jóvenes universitarios de otros países, como el caso de Chile, México y Perú, se evidencia un alto porcentaje de asistencia.

Estos estudios están más enfocados a metodologías cuantitativas, dejando de lado los métodos cualitativos, que permiten evidenciar la motivación y la preferencia del consumo de prácticas culturales entre los jóvenes universitarios, lo que impide reflexionar sobre el papel de la universidad como promotora de una educación y bienestar integral de los futuros profesionales.

En cuanto a nivel teórico y metodológico, los estudios están relacionados con las Ciencias Sociales, en las que las características sociodemográficas establecen patrones frente a sus gustos y preferencias por las actividades que desarrollan los estudiantes dentro y fuera de la universidad. 
Las investigaciones encontradas solo se basan en el consumo cultural de los estudiantes, y dejan por fuera a otros actores de gran importancia, como los docentes y administrativos, quienes juegan un papel significativo dentro de la comunidad universitaria.

Los autores en sus investigaciones llaman la atención sobre cómo el consumo de la tecnología se ha elevado y diversificado con los diferentes servicios que brinda la Internet, como las redes sociales, Whatsapp, Messenger, entre otros, los cuales han transformado las prácticas culturales de los universitarios, creando nuevos patrones de consumo cultural, de reconocimiento, de identidad, de sentido y de significado en sus comportamientos sociales y simbólicos. Los estudios realizados en ciudades como Medellín, Villavicencio y Bogotá muestran que un alto porcentaje de la población dedica su tiempo al uso de las TICs, ya sea para consulta académica, o para jugar, comprar, escuchar y descargar música, ver películas o interactuar en las redes sociales.

Estos cambios culturales están modelando a profundidad la vida de los universitarios y de sus inacabables posibilidades de formación. Como lo plantea García Canclini (2006), hay una relación entre consumo y ciudadanía y, sobre todo, no podemos olvidar que "el consumo sirve para pensar", para establecer nuevas políticas frente a las actividades que se realizan dentro y fuera de las instituciones educativas sobre el aprovechamiento del tiempo libre y como complemento de las actividades académicas.

Finalmente, las investigaciones consultadas y analizadas mostraron que las prácticas culturales entre universitarios están cambiando, y se está produciendo un consumo masivo de tecnologías de la información y la comunicación, lo cual está transformando la vida sociocultural de los jóvenes.

\section{Referencias}

Acosta, K., Espinoza, K., Palacios, P., Precich, A., Torres, V., y Arellano, A. (2014). Radio cultural: estudio del consumo que los estudiantes y trabajadores de la Universidad de Colima tienen de Universo 94.9. Recuperado de http://w w.ucol.mx/interpretextos/pdfs/781_inpret1114.pdf

Aguilar, J., y Ramírez N. (2007). Hábitos de consumo de las tecnologias de la información en los estudiantes universitarios de Tijuana-México. Recuperado de https://mpra.ub.uni-muenchen.de/4718/1/MPRA_paper_4718.pdf

Alarcón, D., y Larraz, I. (2015). Conozca a los 'Millennials', ¿la generación que salvará al planeta? El Tiempo. Recuperado de https://www.eltiempo.com/archivo/documento/CMS-15860315

Alata, P. (2013). Lima cómo vamos. Recuperado de http://www.limacomovamos.org/cm/wp-content/uploads/2014 /03/EncuestaLimaComoVamos2013.pdf

Andrade, D. (2016). Determinantes de la asistencia a museos en Colombia. Una perspectiva desde la economia de la cultura (tesis doctoral). Universidad de Sevilla, Sevilla, España.

Andrade, M. C. (2007). La lectura en los universitarios. Un caso específico: Universidad Colegio Mayor de Cundinamarca, Colombia. Tabula Rasa, (7), 231-249.

Antoine-Faúndez, C., y Carmona-Jiménez, J. (2015). Museos y jóvenes: entre la incomprensión y el desencanto. Percepciones juveniles sobre el consumo cultural de museos en Chile. Arte, Individuo y Sociedad, 27(2), 227-242.

Arango, G., y González, M. (2009). Televidencias juveniles en Colombia: fragmentación generada por un consumo multicanal. Palabra Clave, 12(2), 215-234.

Arroyo, G. (2011). Consumo cultural en jóvenes estudiantes de pregrado de la Pontificia Universidad Católica del Perú (tesis de pregrado). Pontificia Universidad Católica del Perú, Lima, Perú. Recuperado de http://tesis.pucp.edu. pe/repositorio/handle/123456789/1166

Barrios, A. (2009). Los jóvenes y la red: usos y consumos de los nuevos medios en la sociedad de la información y la comunicación. Signo y Pensamiento, (54), 265-275.

Basantes, A., Naranjo, M., Gallegos, M., Grijalva, P., y Villarreal, J. (2016). Hábitos de consumo y uso de medios digitales en los estudiantes de la Universidad Técnica del Norte. Ecos de la Academia, (4), 33-41. Recuperado de https:/ /issuu.com/utnuniversity/docs/ecos-n04

Bourdieu, P. (1998). La Distinción. Criterio y bases sociales del gusto. Madrid: Editorial Taurus. 
Cabanzo, C. (2009). Ocio y tiempo libre: una aproximación a sus representaciones y percepciones en la Universidad Pedagógica Nacional. Recuperado de http://www.ascodes.com/wp-content/uploads/2017/11/OCIO_Y_TIE MPO_LIBRE.pdf

Cabrera, C., Haro, L., Orozco, M., Tornero, C., Báez, L., y Zavala, M. (2017). Prevalencia del consumo cultural, del tiempo libre y de medios de comunicación, en estudiantes de ciencias de la salud en dos universidades latinoamericanas: Guadalajara y Guatemala. Recuperado de http://www.medigraphic.com/pdfs/saljalisco/sj-2 017/sj171e.pdf

Callejas, E. (2016). Estilos de vida Universitaria de los estudiantes de la Universidad Mayor de San Andrés, Bolivia. Educación Superior, 1(1), 31-41.

Caro, D., y Vásquez, A. (2012). Consumo cultural y uso del tiempo libre en universitarios del área metropolitana. En Clave Social, 1(2), 8-19.

Casillas, M., Molina A., y Colorado, A. (2011, noviembre). Usos del tiempo de los estudiantes universitarios. Ponencia presentada en XI Congreso Nacional de Investigación Educativa, México D.F. Recuperado de http://www.com ie.org.mx/congreso/memoriaelectronica/v11/docs/area_16/1087.pdf

Castells, M. (2000). Globalización, sociedad y politica en la era de la Información. Recuperado de https://dialnet.uniri oja.es/descarga/articulo/4008342.pdf recuperado 2 de mayo 2018.

Castells, M. (2007). Comunicación móvil y sociedad. Una perspectiva global. Recuperado de http://www.eumed.net/li brosgratis/2007c/312/comunicacion\%20movil\%20segun\%20el\%20genero.htm

Chaparro, H., y Guzmán, C. (2017). Jóvenes y consumo cultural. Una aproximación a la significación de los aportes mediáticos en la preferencia juvenil. Anagramas, 15(30), 121-142.

Consejo Nacional para la Cultura y las Artes México (Conaculta). (2006). Encuesta Nacional de Lectura en la población mexicana en donde se incluyen jóvenes universitarios México D.F. Recuperado de http://sic.cultura.gob.mx/encu esta/Encuesta\%20de\%20Lectura\%20ok.pdf

Dávila, M., Rivera, R., y Chávez, R. (2017, noviembre). Usos del tiempo y consumo cultural: Caracterización de estudiantes de una Universidad Rural del Sur del Estado de Sonora. Ponencia presentada en XIV Congreso Nacional de Investigación Educativa, San Luis Potosí. Recuperado de http://www.comie.org.mx/congreso/me moriaelectronica/v14/doc/2122.pdf

De la Puente, L. (2017). Motivación hacia la lectura, hábito de lectura y comprensión de textos en estudiantes de psicología de dos universidades particulares de Lima Perú. Revista Argumentos, 1(11), 70-75.

Durand, P., Bombelli, E., Barberis, G., y Bouzón, S. (2009). Representaciones y modo de uso de nuevas tecnologías de información y comunicación entre estudiantes universitarios de Buenos Aires. Revista Iberoamericana de Ciencia, Tecnologia y Sociedad - CTS. Recuperado de http://www.revistacts.net/files/Portafolio/durand_editado.pdf

Gamboa A., y Rivera Viera, O. (2015). Jóvenes universitarios: relación con el arte, la cultura y la tecnología en la Universidad Javeriana (tesis de pregrado). Pontificia Universidad Javeriana, Cali, Colombia. Recuperado de http ://vitela.javerianacali.edu.co/bitstream/handle/11522/3891/Jovenes_universitarios_relacion.pdf?sequence $=1$

García Canclini, N. (2006). El consumo cultural: una propuesta teórica. En G. Sunkel (coord.), El Consumo Cultural en América Latina. Colombia (pp. 72-95). Bogotá, Colombia: Convenio Andrés Bello.

Gilardoni, S. (2006). Valoración del libro y mecanismos de acercamiento a la lectura en los estudiantes universitarios Chile. Recuperado de http://eprints.rclis.org/8083/1/erie_16.pdf

Gómez, S., y Riverón, K. (2017). Prácticas Culturales en jóvenes universitarios Cubanos. Trilogía Ciencia Tecnología Sociedad, 9(16), 101-111. https://doi.org/10.22430/21457778.177

Hinojosa L. (2012). Consumo y prácticas culturales de la comunidad universitaria de una universidad mexicana [Universidad Autónoma de Nuevo León México]. Recuperado de http://www.aeic2012tarragona.org/comunica cions_cd/ok/58.pdf

Huertas, C., Caro, D., Vásquez, A., y Vélez, J. (2008). Consumo cultural y uso del tiempo libre en estudiantes lasallistas. Revista Lasallista de Investigación, 5(2), 36-47. 
Juni, M. (2015). Consumo musical de los jóvenes uruguayos (tesis de pregrado). Universidad de la República, Montevideo, Uruguay. Recuperado de https://www.colibri.udelar.edu.uy/jspui/bitstream/20.500.12008/1000 8/1/TS_JuniMaida.pdf

Marcano, B. (2012). Características sociológicas de videojugadores online y el e-sport. El caso de Call of duty. Pedagogía Social. Revista Interuniversitaria, (19), 113-124.

Munguía, P. (2006). Consumo de medios de comunicación y la construcción de la imagen de los partidos politicos en jóvenes Universitarios (tesis de pregrado). Universidad Nacional Mayor de San Marcos, Lima, Perú. Recuperado de htt p://cybertesis.unmsm.edu.pe/handle/cybertesis/1290

Nivón, E., y Sánchez, D. (2012). Algunas consideraciones sobre los estudios de consumo cultural en México y en Chile. Alteridades, 22(44), 59-79.

Nobles, D., Londoño, L., Martínez, S., Ramos, A., Santa, G., y Cotes, A. (2016). Tecnologías de la comunicación y relaciones interpersonales en jóvenes universitarios Universidad Simón Bolívar Sede Barranquilla. Educación y Humanismo, 18(39), 14-27.

Ortega, F. (2012). El impacto de las nuevas tecnologías digitales en la cultura comunicativa de los jóvenes universitarios. Una visión panameña. Revista Communifé, 12(12), 73-87.

Paredes, M., y Behn, V. (2008). Utilización del tiempo libre de estudiantes del área de Salud de la Institución Educacional Santo Tomas, Talca - Chile. Ciencia y Enfermería, 14(1), 53-62.

Pérez, Y., y Castro, W. (2015). Jóvenes en la encrucijada de los nuevos tiempos. Una mirada a sus consumos culturales Universidad Granma - Cuba. Revista Cientifica Guillermo de Ockham, 13(1), 127-134.

Portocarrero, M. (2017). Estudio comparativo sobre el consumo cultural de los universitarios de la Universidad Nacional Mayor de San Marcosy la Universidad San Martin de Porres en Perú (tesis de maestría). Universidad César Vallejo, Trujillo, Perú. Recuperado de http://repositorio.ucv.edu.pe/bitstream/handle/UCV/9016/Portocarrero_PM. pdf?seuence $=1$

Puerta D., y Carbonell R. (2013). Uso problemático de Internet en una muestra de estudiantes universitarios colombianos. Avances en Psicología Latinoamericana, 31(3), 620-631.

Quintar A., y Borello J. (2014). Consumos culturales en Argentina: El caso del cine en la población de estudiantes universitarios. Imagofagia, (9), 1-21.

Rangel, L., y Ochoa, M. (2012). El tiempo libre de los estudiantes universitarios de la Facultad de Comercio de Tampico. CienciaUAT, 6(3), 20-26.

Ravettino, A. (2012). Prácticas de lectura. Acerca del placer y la literatura en jóvenes adultos estudiantes. Recuperado de http://dspace.uces.edu.ar:8180/xmlui/bitstream/handle/123456789/2352/Practica_Revettino_Destefanis .pdf?sequence $=1$

Ríos, J., Matas, A., y Gómez, E. (2011). Investigación sobre el uso de la televisión por estudiantes, de educación, de universidades hispanoamericanas. Profesorado, 15(1), 305-323.

Rodríguez, A., Solano E., Martínez, A., y del Villar, L. (2013). Lectura y Escritura en la Universidad Colombiana. Zona Próxima, (18), 2-17.

Rodríguez, M. (2018). Redes sociales y hábitos de consumo en estudiantes universitarios, caso Universidad Pedagógica y Tecnológica de Colombia. Revista Espacios, 39(9), recuperado de http://www.revistaespacios.com/a18v39n0 9/a18v39n09p37.pdf

Sánchez, C. (2013). Prácticas de consumo audiovisual en internet en estudiantes universitarios de la ciudad de Medellín (Upb, Udea, Politécnico Jic, Udem, Eafit) (tesis de doctorado). Universidad Pontificia Bolivariana, Medellín, Colombia.

Sandoval, N. (2016). Diagnóstico acerca del uso del ocio y el tiempo libre entre los estudiantes de la Universidad Nacional Experimental del Táchira, Pedagogía Social. Revista Interuniversitaria, (30), 169-188.

Suing, A., Ordóñez K., y Ortiz, C. (2018). El consumo de radio y televisión de los estudiantes universitarios del Ecuador [mensaje en un blog]. Recuperado de https://abelsuing.wordpress.com/2018/01/19/el-consumo-de-radio-y-tel evision-de-los-estudiantes-universitarios-del-ecuador/ 
Terrazas, F., Lorenzo, O., y González, P. (2013). Consumo musical de estudiantes universitarios de México. Una comparación entre alumnos de distintas facultades de una universidad mexicana. Revista Electrónica de LEEME, (32), 121-134.

Velásquez, B. (2015). Consumo y uso de medios de comunicación de jóvenes universitarios ecuatorianos en tiempos de convergencia (tesis doctoral). Universidad de Santiago de Compostela, Santiago de Compostela, Chile. Recuperado de https://minerva.usc.es/xmlui/handle/10347/15340

Vergara, M., Cerezo, M., Cifuentes O., Nieto, E., y Parra, J. (2009). Consumo de medios masivos de comunicación en estudiantes universitarios de Manizales. Revista Hacia la Promoción de la Salud, 14(1), 124-138.

Warnier, J. P. (2001). La mundialización de la cultura. Quito: Ediciones Abya-Yala.

Yuquilima, M. (2015). Estudio del insuficiente hábito en la lectura de los estudiantes del primer semestre de la carrera de Comunicación Social de la Universidad de Guayaquil, periodo 2014-2015 (tesis de pregrado). Universidad de Guayaquil, Guayaquil, Ecuador. Recuperado de http://repositorio.ug.edu.ec/handle/redug/7484

\section{Notas}

* Artículo de revisión.Este artículo de revisión es parte de los productos parciales del proyecto "Consumo de prácticas culturales en la Universidad de Boyacá" desarrollado en el Grupo de Investigación Ethos de la Facultad de Ciencias Humanas y Educativas realizado por las docentes - investigadoras de la Universidad de Boyacá, Mg. Clara Inés Villamil Guzmán y Mg. Luz Marina Hurtado Torres, y adscritas al Departamento de Ética y Humanidades.

\section{Licencia Creative Commons CC BY 4.0}

Cómo citar este artículo: Villamil Guzmán, C. I., y Hurtado Torres, L. M. (2019). Consumo de prácticas culturales en jóvenes universitarios de algunos países latinoamericanos. Signo y Pensamiento, 38(75). https: //doi.org/10.11144/Javeriana.syp38-75.cpcj 